\section{International guidelines for \\ contrast-enhanced ultrasonography: ultrasound imaging in the new millennium}

\author{
Christian Pállson Nolsøe, Torben Lorentzen \\ Ultrasound Section, Department of Gastric Surgery, Herlev Hospital, University of \\ Copenhagen, Herlev, Denmark
}

The intent of this review is to discuss and comment on common clinical scenarios in which contrast-enhanced ultrasonography (CEUS) may play a decisive role and to illustrate important points with typical cases. With the advent of CEUS, the scope of indications for ultrasonography has been dramatically extended, and now includes functional imaging and tissue characterization, which in many cases enable tumor diagnosis without a biopsy. It is virtually impossible to imagine the practice of modern medicine as we know it in high-income countries without the use of imaging, and yet, an estimated two thirds of the global population may receive no such care. Ultrasound imaging with CEUS has the potential to correct this inequity.

Keywords: Abdomen; Image enhancement; Ultrasonography; Contrast media; Microbubbles

\section{Introduction}

This review deals with the four sets of international contrast-enhanced ultrasonography (CEUS) guidelines that have been published from 2004 to 2013 [1-5] and discusses the clinical implications thereof, with an emphasis on daily clinical work. The intention is to focus on common clinical scenarios in which CEUS plays a decisive role and to illustrate these scenarios with typical cases. According to the World Health Organization (WHO), two thirds of the world's population has no access to any kind of medical imaging [6]. Ultrasonography, including refinements such as elastography and CEUS, has tremendous potential to rectify this inequity [7]. We discuss this possibility in light of four sections in which we comment on each set of guidelines separately with regard to their most important aspects. In addition, pictorial explanations drawing on clinical cases are provided to emphasize selected points.

\section{Guidelines for the Use of Contrast Agents in Ultrasonography}

The first generation of international CEUS guidelines was issued in 2004 by the European Federation of Societies for Ultrasound in Medicine and Biology (EFSUMB) [1]. This article was important for two major reasons. First, it presented a scientific endorsement of the clinical use of contrast agents during ultrasonography as a decisive imaging modality in the work-up of focal liver lesions (FLLs)

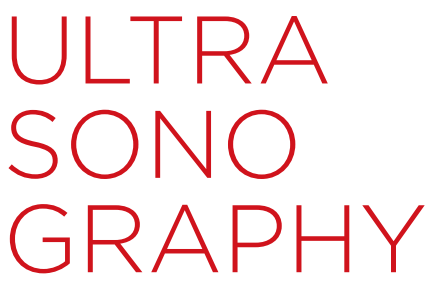

REVIEW ARTICLE

http://dx.doi.org/10.14366/usg. 15057 pISSN: 2288-5919 • elSSN: 2288-5943

Ultrasonography 2016;35:89-103

Received: September 15, 2015

Revised: December 23, 2015

Accepted: December 23, 2015

Correspondence to:

Christian Pállson Nolsøe, MD, PhD,

Ultrasound Section, Department

of Gastric Surgery, Herlev Hospital,

University of Copenhagen, Herlev

Ringvej 75, 2730 Herlev, Denmark

Tel. $+45-38683087$

Fax. +45-38144189

E-mail: cnolsoe@dadlnet.dk

This is an Open Access article distributed under the terms of the Creative Commons Attribution NonCommercial License (http://creativecommons.org/ licenses/by-nc/3.0/) which permits unrestricted noncommercial use distribution, and reproduction in any medium, provided the original work is properly cited.

Copyright @ 2016 Korean Society of Ultrasound in Medicine (KSUM)

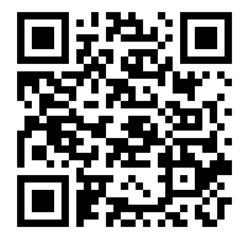

How to cite this article:

Nolsøe CP, Lorentzen T. International guidelines for contrast-enhanced ultrasonography: ultrasound imaging in the new millennium. Ultrasonography. 2016 Apr;35(2): 89-103. 
and as an ideal tool for all phases of liver tumor ablation. Second, it established the acronym of CEUS to describe this technique. A search on Scopus performed on August 28, 2015 showed that the article had been cited 308 times in 135 different journals.

These guidelines provided a detailed explanation of how to detect and characterize liver tumors and how to differentiate among various benign entities based on the enhancement pattern after the administration of the contrast agents, and demonstrated that while some refinements may be necessary, a simple algorithm can be used for the most common findings (Table 1). The main criterion for malignancy on CEUS is contrast hypo-enhancement in the late phase, whereas benign lesions typically remain isoenhancing to hyperenhancing in comparison with the surrounding normal liver tissue. Benign entities can reliably be differentiated from each other by carefully scrutinizing the arterial contrast uptake phase; for instance, a globular filling-in pattern is typical for hemangioma and, along with continued late-phase enhancement, is pathognomonic for this diagnosis.

FLLs represent a very common incidental finding in modern medicine due to the frequent use of cross-sectional imaging in most high-income countries. This comes as no surprise to anyone familiar with abdominal ultrasonography. It may, however, be surprising to discover that despite the frequency of this finding, the literature contains no prospective data from clinically controlled studies describing the frequency and characterization of sonographically detected incidental FLLs. In a retrospective computed tomography (CT) study, Volk et al. [8] evaluated 1,892 examinations from a single institution that were performed for various reasons, but specifically without previously known or suspected FLL, liver cirrhosis, or underlying malignant disease. They identified a total of 100 cases with unexpected FLL, and a CT diagnosis of incidentally discovered benign liver lesions was made in $33 \%$ of those 100 cases [8]. In contrast, a somewhat surprising $17 \%$ risk of malignancy was found by Little et al. [9] in a study of 64 patients admitted over a 5-year period to a surgical referral department for the diagnostic work-up of solid liver lesions incidentally diagnosed at scanning of otherwise "relatively well" patients. The truth most likely lies somewhere between these scenarios; and in daily clinical practice FLLs may be encountered either incidentally or in follow-up scans of oncology patients. In any case, the basic questions that arise are identical: "Are the lesions benign or malignant?" and "If the lesion is malignant, then what kind of malignancy is it?"

Diagnostic ultrasonography has several important qualities that cannot be equally or easily achieved using CT or magnetic resonance imaging (MRI). Among these are features crucial to the two questions above, including spatial and, in particular, temporal resolution, as well as the practical aspects of real-time examinations and scanner availability. In three different European multicenter studies, CEUS was found to be comparable to contrast-enhanced CT or MRI [10-16]. The most extensive material from the German Ultrasound Society drew on 1,349 patients and reported a positive predictive value of $95.4 \%$ and a negative predictive value of $95.7 \%$ in differentiating between malignant and benign lesions [10]. In two 2011 meta-analyses of CEUS versus contrast-enhanced CT and $\mathrm{MRI}$, the overall performance of the three modalities was statistically equal, with reported sensitivity and specificity ratios ranging from $81 \%$ to $89 \%$. Interestingly, however, CEUS had the highest diagnostic odds ratio $[17,18]$.

In the most recently published systematic review from the National Institute for Health Research of the United Kingdom [19], which identified a total of 854 references and included 21 studies, Westwood et al. [19] concluded that SonoVue (Bracco, Milan, Italy) CEUS can provide similar diagnostic performance to contrastenhanced CT and MRI for the major clinical applications: (1) the characterization of FLLs detected in the sonographic surveillance of cirrhosis patients, (2) the detection of liver metastases in patients with colorectal cancer, and (3) the characterization of incidentally detected FLLs. They made a strong statement that deserves the

Table 1. Algorithm for using contrast-enhanced ultrasonography to characterize focal liver lesions based on the enhancement pattern after the administration of contrast agents

\begin{tabular}{|c|c|c|c|c|}
\hline & Type & Arterial phase $(10-20 \mathrm{sec})$ & Portal phase (30-45 sec) & Late phase (>120 sec) \\
\hline \multirow[t]{3}{*}{ Benign } & Hemangioma & Globular enhancement & Centripetal filling & Iso-/hyper-enhancement \\
\hline & Focal nodular hyperplasia & $\begin{array}{l}\text { Hyper-enhancement spoke wheel } \\
\text { appearance centrifugal filling }\end{array}$ & $\begin{array}{l}\text { Moderately hyper-/ } \\
\text { iso-enhancement }\end{array}$ & Iso-enhancement scar (40\%) \\
\hline & Hepatocellular adenoma & Hyper-enhancement & Transition & Iso-enhancement \\
\hline \multirow[t]{3}{*}{ Malignant } & Hepatocellular carcinoma & Hyper-enhancement & Iso-/slightly hypo-enhancement & Hypo-enhancement \\
\hline & Metastasis, hypervascular & $\begin{array}{l}\text { Hyper-enhancement with/ } \\
\text { without central necrosis }\end{array}$ & Iso-/slightly hypo-enhancement & Strong hypo-enhancement \\
\hline & Metastasis, hypovascular & No enhancement or peripheral rim & Iso-/slightly hypo-enhancement & Strong hypo-enhancement \\
\hline
\end{tabular}

Time ranges in the parentheses are the delayed time for each imaging after contrast agent injection. 
full attention of medical professionals working in this field: "If the main use of liver imaging is considered to be the rapid ruleout of malignancy, equivalent diagnostic performance may be sufficient for SonoVue CEUS to be preferred over other imaging modalities. A potential advantage of using SonoVue CEUS would be the option of completing the assessment at the same time as the initial unenhanced ultrasonography." These conclusions are broadly supported [20-22] and elegantly framed by Friedrich-Rust et al. [23] in the following statement: "Use CEUS as first method of choice for the diagnostic work up of focal liver lesions if B-mode and Dopplerultrasonography are not conclusive."

In addition, CEUS has an excellent safety profile [24] and patient compliance, as well as no issues regarding organ function or radiation exposure. Therefore, when available, CEUS should be the first method to answer the above question of "Are the lesions benign or malignant?" However, CEUS cannot provide a reliable answer to the question "If the lesion is malignant, then what kind of malignancy is it?" If a histological diagnosis is deemed necessary for treatment planning, a tissue sample must be obtained, in which case an ultrasound-guided biopsy is the obvious choice [25]. Fig. 1 (with Video clips 1-3) provides a pictorial illustration of this concept of the clinical work-up of newly diagnosed liver lesions if the scenario includes the suspicion of malignant disease. In the case of malignancy, a standard protocol for staging and treatment planning should always include full-body imaging modalities such as contrast-enhanced CT or MRI according to the specific malignancy

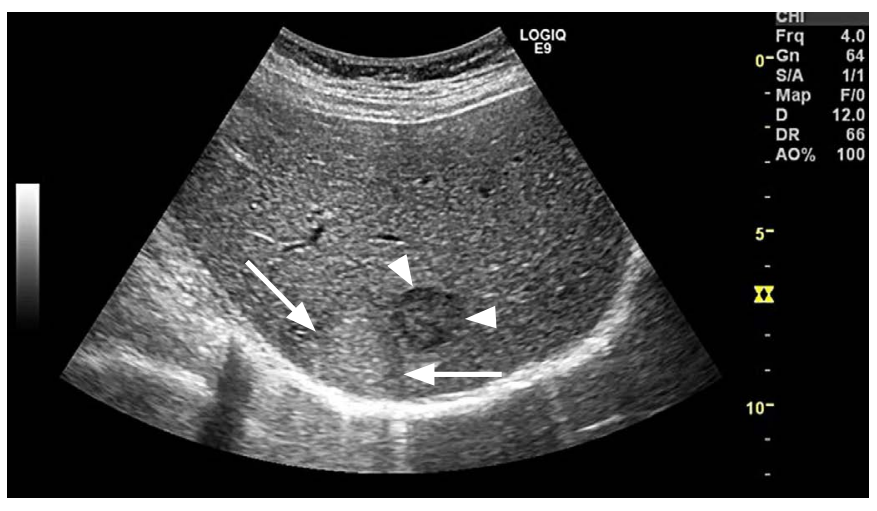

A

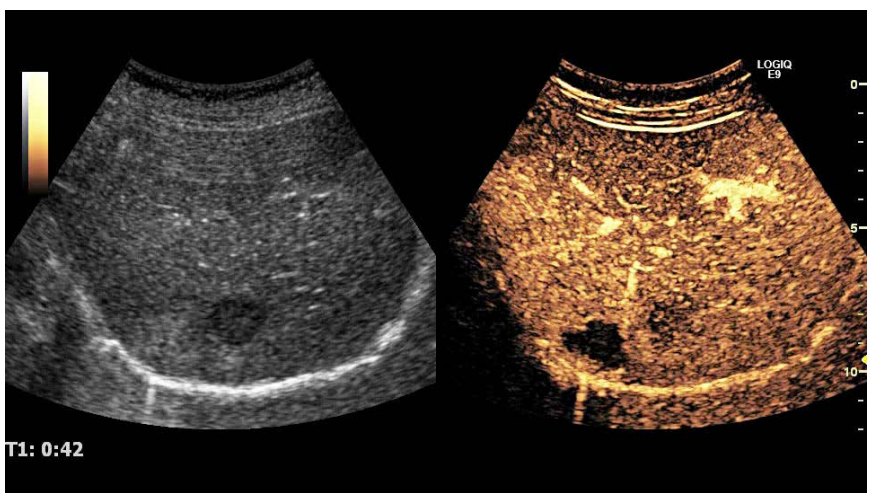

C

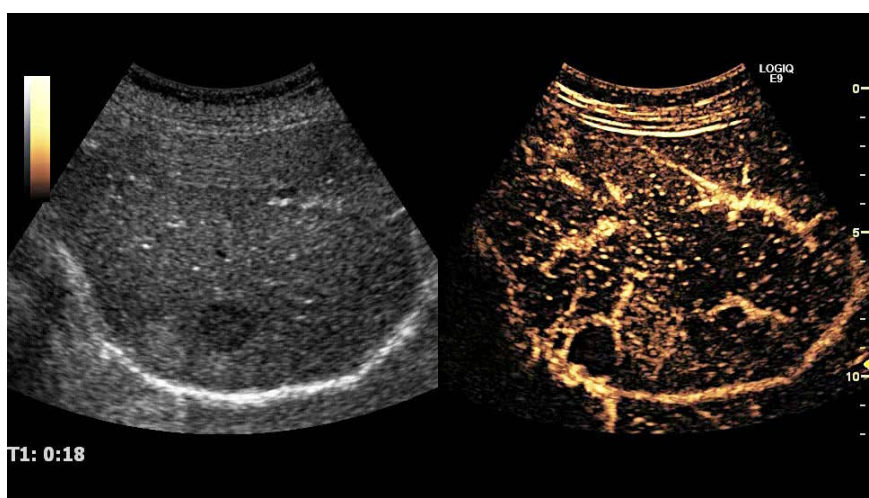

B

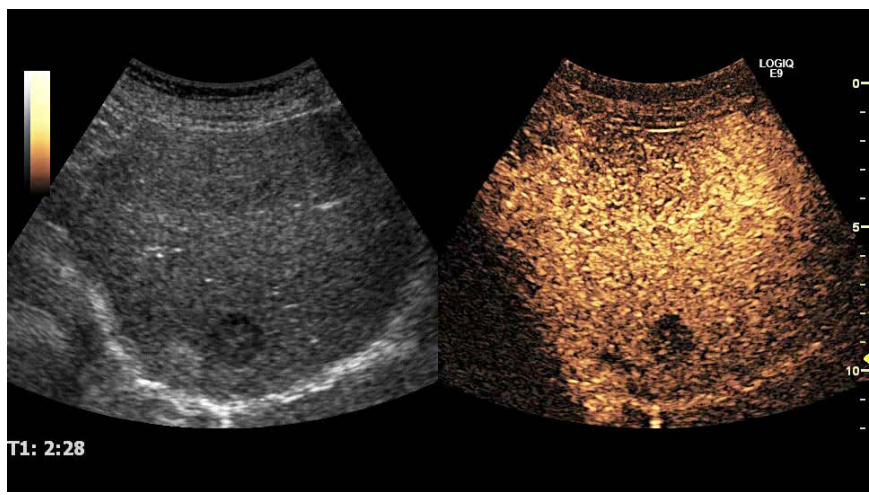

D

Fig. 1. A 60-year-old woman referred for an ultrasonography to rule out gallstones incidentally diagnosed with two focal solid lesions in the right liver lobe located laterally in segment 6 and very closely separated.

A. The two lesions show different appearances on B-mode imaging; one is hyperechoic (arrows) with slightly posterior enhancement and peripheral lie and the other is echo-poor with a halo around it (arrowheads). From the B-mode imaging alone, one would suspect the echorich lesion to be a hemangioma and the echo-poor lesion to be a metastasis. A routine contrast-enhanced ultrasonography was performed to further characterize these lesions. B-D. The hyperechoic lesion shows globular filling-in contrast enhancement, whereas the hypoechoic lesion demonstrates arterial hyperenhancement (B) and contrast wash-out pattern on portal (C) and late phase (D) images, thus confirming the B-mode diagnosis of hemangioma and metastasis. Since the patient had no known primary cancer, the institutional tumor board decided that an ultrasound-guided biopsy was indicated, and a TruCut 1.2-mm core biopsy provided the diagnosis of metastatic hepatic adenocarcinoma of colorectal origin. 


\section{in question.}

A more common problem, however, is incidentally diagnosed, but sonographically benign liver lesions and the further clinical work-up, if any, of such lesions. In other words, the question is how to handle the unexpected finding of an FLL that, both sonographically and with respect to the patient's symptoms and medical record, is deemed a benign finding. Here, as in many other times in life, a good piece of advice is to "keep it simple," and the

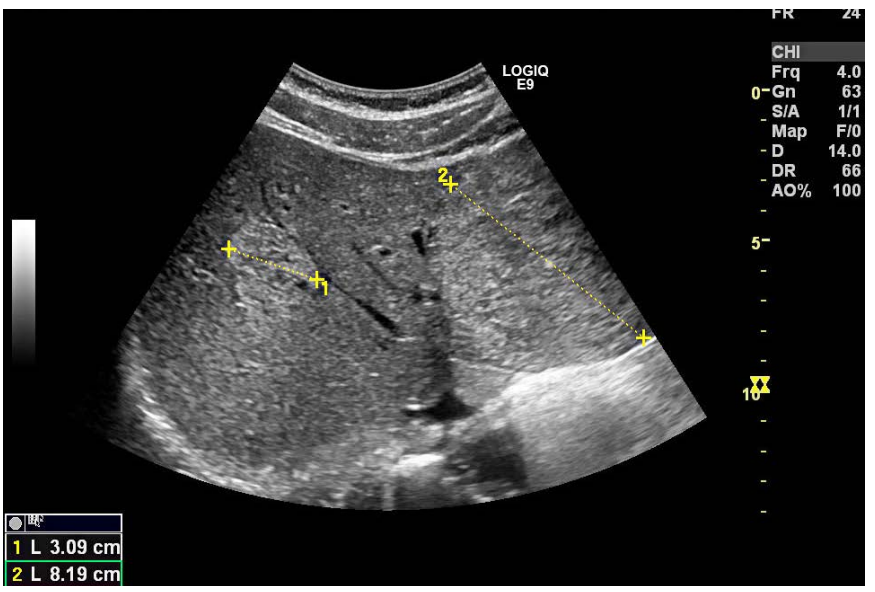

A

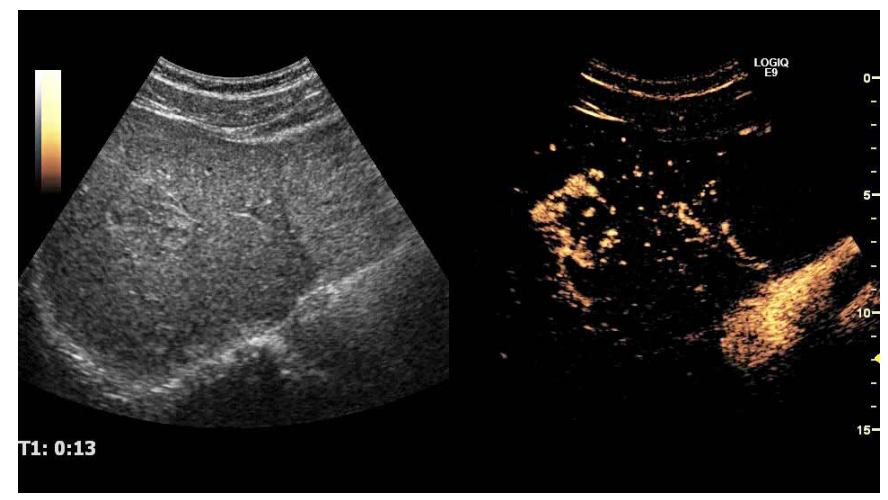

C

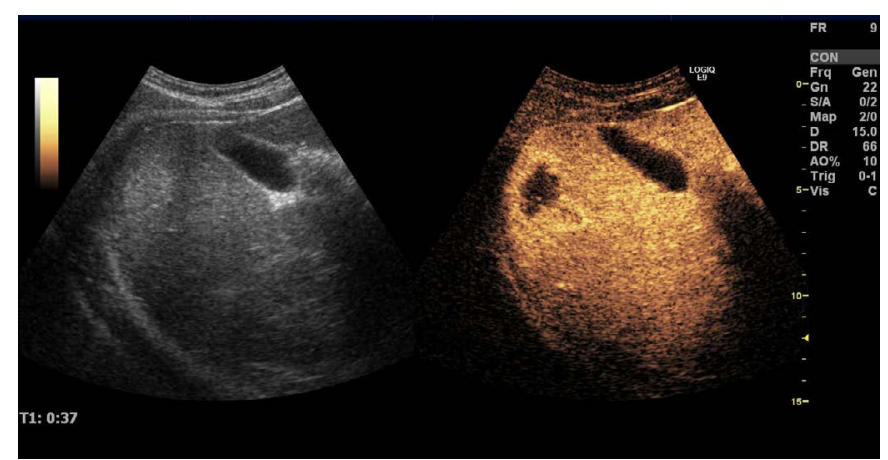

E simplest rule one can apply is to follow the guidelines. The current guidelines are identical to the 2004 guidelines with regard to this issue, and state that CEUS is indicated for "incidental findings on routine ultrasonography." This recommendation makes no further differentiation, and no doubt is left as to whether it is indicated in all cases, including the typical benign B-mode appearance. In the scenario of an incidentally detected lesion, this means that, unless the lesion is a sonographically typical cyst, a CEUS exam should be

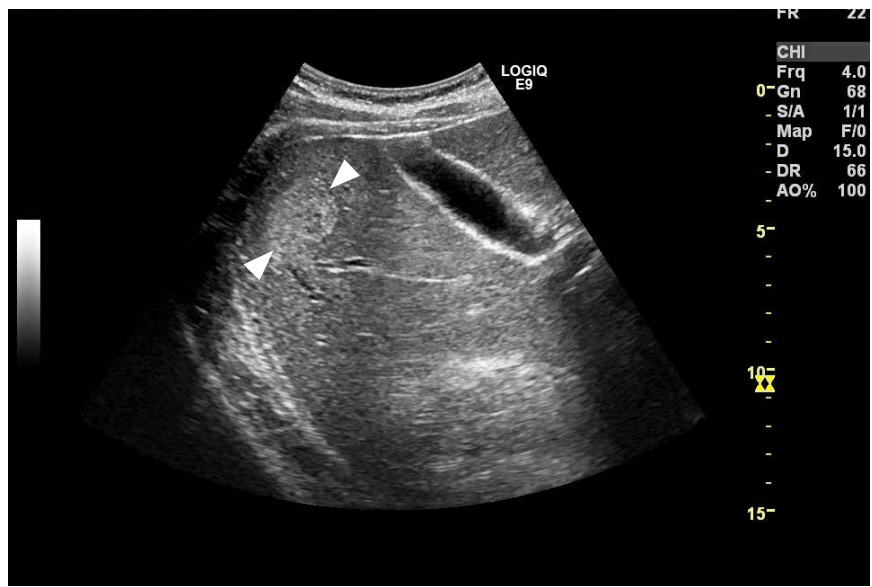

B

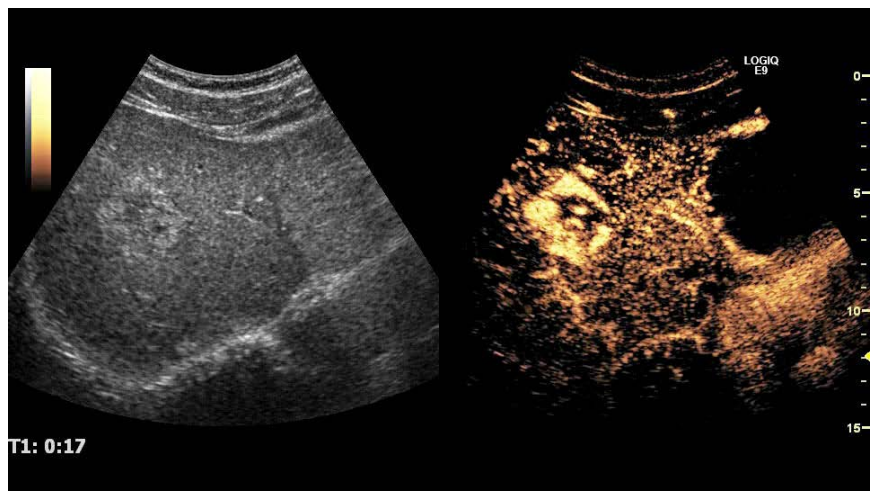

D

Fig. 2. A 53-year-old man with no history or clinical suspicion of cancer referred to rule out gallstones.

A. B-mode transverse sonogram shows two hyperechoic lesions (between crosshairs) in both lobes of the liver. B. Another hyperechoic lesion (arrowheads) is defined in lower section of right hepatic lobe. C-E. Contrast-enhanced ultrasonography (CEUS) sequences illustrate arterial contrast uptake with very early (before normal liver tissue) enhancement of the centrally located lesion with a centrifugal pattern, a feeding artery consistent with the focal nodular hyperplasia (FNH) arterial pattern, and the slower centripetal globular filling-in enhancement pattern of the other two lesions (one in each liver lobe), consistent with the arterial pattern of hemangioma. 


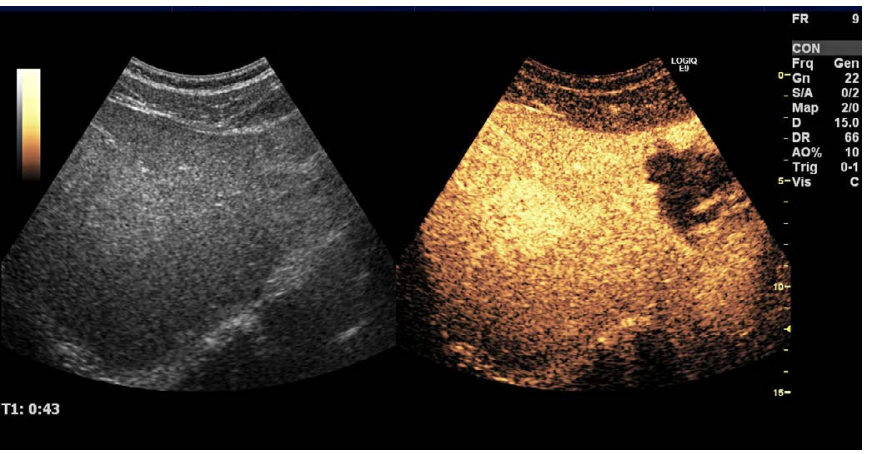

$\mathrm{F}$

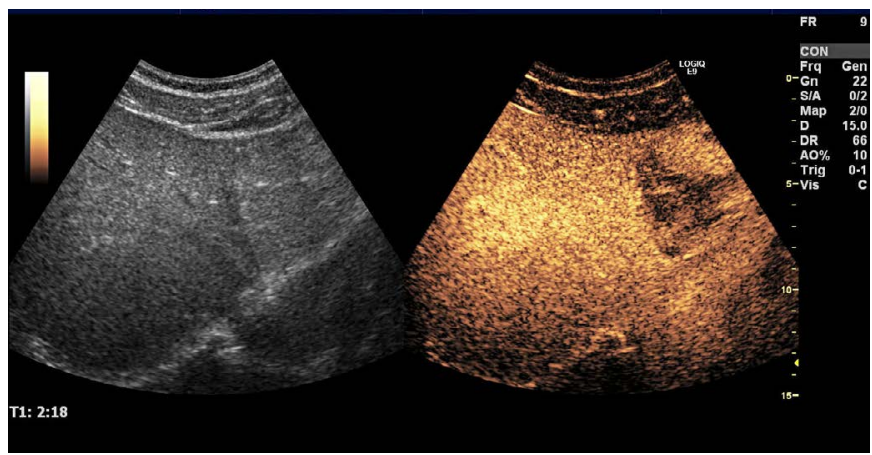

G

Fig. 2. F. CEUS during the portal phase shows sustained hyperenhancement of FNH and typical slow filling-in of a large hemangioma. G. CEUS during the parenchymal phase still shows sustained hyperenhancement of FNH and typical slow filling-in of a large hemangioma in which the central areas do not display enhancement due to necrosis and scarring with both features extending long into the parenchymal phase. The patient subsequently underwent contrast-enhanced magnetic resonance imaging to confirm the benign nature of all lesions with a 1-year clinical follow-up. In the work-up of presumed benign focal liver lesions this pattern is unusual, but it is nonetheless important to be aware of the fact that in daily practice, multiple simultaneous hemangiomas are frequently encountered and their coexistence with FNH is seen more often than would be expected from pure chance [9].

performed. This is the simplest and safest rule to follow, and it is also exactly what the guidelines have recommended from the first set of guidelines issued in 2004 to the latest guidelines issued in 2012. If one follows this simple rule, it is not necessary to establish complicated algorithms to determine whether an incidental FLL should be further characterized on B-mode ultrasonography in order to reach a final decision or if a CEUS study should be next step.

However, many experienced ultrasonography diagnosticians, including myself, feel that there is an issue with hemangiomas and that this issue deserves further commentary. Hemangioma is the most common tumorous condition of the liver and was found in an autopsy study [26] to be present in no less than $20 \%$ of men, although with a mean size of $5.2 \mathrm{~mm}$, meaning that many of them were very small and most likely would have remained undiscovered on routine ultrasonography. In the abovementioned study of patients referred for further assessment [9], hemangiomas constituted $52 \%$ of the lesions. Hemangioma is also the most common benign tumor and the most common echo-rich tumor of the liver [27]. Most hemangiomas have very typical B-mode features, including a welldemarcated, round, and uniformly echo-rich appearance with a usually peripheral lie, posterior enhancement, and a diameter less than $3 \mathrm{~cm}$. Provided that the tumor is detected in a patient without any history of cancer or suspicion thereof, a diagnosis of benignity can be established using conventional ultrasonography with an estimated probability of $>95 \%$, and no further imaging is indicated $[20,21,27]$. The definitive characterization of every incidentally found hemangioma with CEUS would lead to a substantial workload and disproportionate cost. Moreover, all such efforts would be in vain because, according to Dietrich et al. [21], neither CEUS nor any other imaging modality would provide the correct diagnosis with a higher certainty than the ultrasonography techniques already available [9]. In approximately $30 \%$ of cases, however, hemangiomas demonstrate atypical sonographic features, and a CEUS study should be the next step for further characterization and to rule out malignancy. The same is true in the clinical scenario where a B-mode exam demonstrates an apparently typical hemangioma in an oncological patient or a patient suspected of malignancy. In such cases, CEUS is also the recommended next step. Fig. 2 (with Video clips 4-7) shows a case with a coincidental diagnosis of multiple simultaneous large hemangiomas in coexistence with focal nodular hyperplasia.

\section{Guidelines and Good Clinical Practice Recommendations for CEUS: 2008 Update}

The second generation of guidelines was published as an article in the February 2008 issue of European Journal of Ultrasound/ Ultraschall in der Medizin [2]. The most important element of this update was the inclusion of extrahepatic applications for CEUS. A search on Scopus on August 28, 2015 showed that the 2008 CEUS guidelines article had been cited a total of 459 times in 139 journals, thus exceeding the citations of the 2004 guidelines by $50 \%$. In addition to the initial indications, this article presented the best-documented newly accepted indications for the use of contrast agents, which were CEUS of the kidney and urinary tract, including the vesico-ureteric reflux, pancreas, and cerebral circulation, and CEUS in abdominal blunt trauma. Guidelines dealing with CEUS of 
liver lesions and CEUS in ablative procedures were updated with the newest data available and the technical aspects were adjusted, but the algorithms for the interpretation of enhancement patterns remained fundamentally unchanged.

One application stands out in comparison with the others, and that is CEUS in blunt abdominal trauma. First, the trauma patient is one of the most commonly encountered clinical scenarios. Second, this new CEUS indication has a tremendous potential for reducing the population-level exposure to ionizing radiation. Third, CEUS for the evaluation of trauma patients has proven beneficial in clinical use on a broad scale. Prior to its inclusion in the 2008 guidelines, only few articles with small study populations were available [28-30]. In recent years, several larger studies have been published confirming that positive outcomes were associated with this technique [31-40], including one prospective multicenter trial by Catalano et al. [41] that enrolled 156 patients with contrastenhanced CT used as the gold standard, and found that per-patient sensitivity increased from $79 \%$ for baseline ultrasonography to 94\% for CEUS. In another study of 256 patients [40] admitted to an emergency department with low-energy blunt abdominal trauma, Sessa et al. [40] found that CEUS had a much higher sensitivity than baseline ultrasonography in the detection of abdominal organ lesions (59\% vs. $96 \%$ ) and in the diagnosis of hemoperitoneum ( $91 \%$ vs. $95 \%)$.

In a pediatric study of 76 children [42] admitted with isolated low-energy abdominal trauma, Menichini et al. [42] reported that the sensitivity and overall accuracy of CEUS in diagnosing solid organ lesions was $100 \%$, as compared to the discouraging values of $39 \%$ and $44 \%$ for ultrasonography alone, with both sets of outcomes evaluated in comparison to contrast-enhanced CT. It was, however, noted that $\mathrm{CT}$ was more accurate than CEUS in identifying prognostic factors such as urinoma and active bleeding.

These conclusions concur perfectly with the recommendations found in the EFSUMB CEUS guidelines on non-hepatic applications published in 2012 [5], which place a special emphasis on being aware of the availability of CEUS as an alternative to CT for patients with low- to moderate-energy blunt abdominal trauma as a supplement to the FAST protocols, especially in the pediatric population and as follow-up imaging modality. However, emphasis was also put on the fact that for high-energy trauma, CT is the modality of choice and that in the case of CEUS-positive findings, contrast-enhanced CT should most likely be performed to exclude active bleeding and obtain a better overall view of the entire abdomen $[40,42]$. Fig. 3 (with Video clip 8) and Fig. 4 (with Video clips 9,10 ) illustrate the CEUS evaluation procedure in trauma cases.

\section{The EFSUMB Guidelines and Recommendations on the Clinical Practice of CEUS: 2011 Update on Non-Hepatic Applications}

This non-hepatic component of the third generation of the CEUS guidelines was published in the February 2012 issue of European

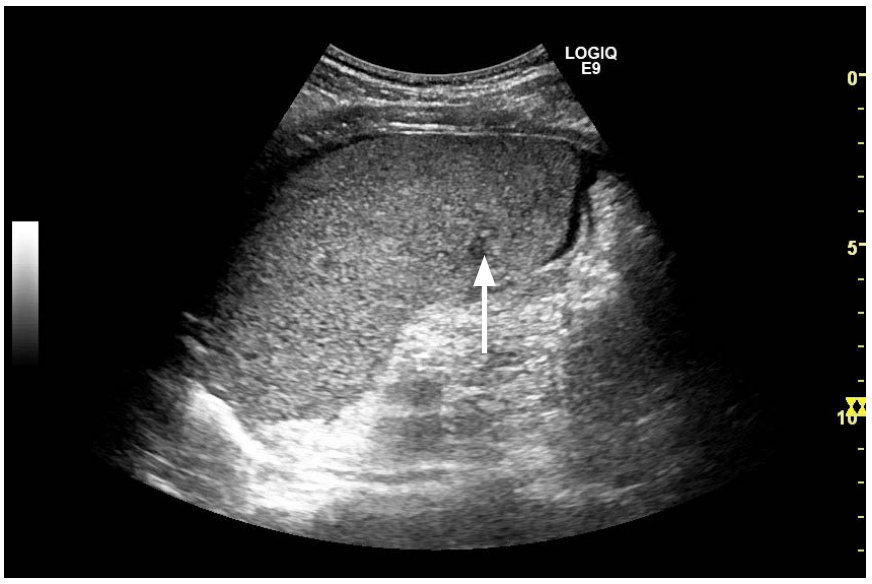

A

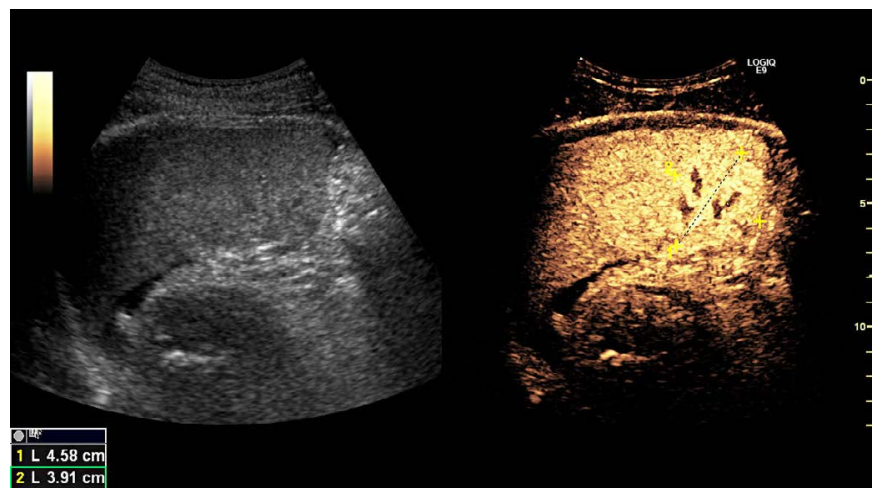

B

Fig. 3. A 26-year-old man referred for 2 days of pain in the left flank after a physical encounter in an ice hockey match.

The patient's condition was stable, his blood pressure was normal, his hemoglobin levels were normal, and he was not in severe pain. The incident was categorized as moderate blunt trauma, and a contrast-enhanced ultrasonography (CEUS) using SonoVue was performed instead of contrast-enhanced computed tomography. A. B-mode ultrasonography in the left flank vaguely gives the impression of a minor intrasplenic hematoma (arrow) and demonstrates a tiny amount of perisplenic fluid collection. B. CEUS of the left flank clearly shows a minor hematoma in the lower pole of the spleen (crosshairs). The patient was treated conservatively and discharged after 2 days. Follow-up, including B-mode imaging and CEUS, showed healing. This figure illustrates the use of CEUS in minor to moderate blunt trauma. 
Journal of Ultrasound/Ultraschall in der Medizin [5]. The key importance of this document was the fact that it placed further emphasis on the inclusion of extra-hepatic applications for CEUS. In addition, each recommendation was graded with respect to type and level of evidence. A search on Scopus on August 28, 2015 showed that the non-hepatic CEUS guidelines had been cited a total of 252 times in 104 different scientific journals.

The diversity of applications covered in this document is huge, and while many ultrasonography professionals may see a range of different clinical entities, no single expert is likely to master the evaluation of all entities. One point that deserves special mention with respect to the clinical use of contrast agents is the application of CEUS in pediatric patients, as it represents a unique medicolegal challenge. This application is controversial and is considered an ethical dilemma by many clinicians. No ultrasound contrast agent has been approved for pediatric use, but in many clinical situations, CEUS could substitute for an alternative imaging modality that would likely include exposure to ionizing radiation or the use of potentially harmful iodinated contrast agents. Most drugs are not specifically approved for pediatric use, but when indicated, most physicians do not hesitate to administer the relevant drug off-label after informed consent from the parents. Indeed, if a necessary medication is not administered out of fear of using an off-label drug, the physician may face serious medico-legal and, it should be added, ethical consequences. Additionally, it should be remembered that most indications mentioned in the non-hepatic guidelines represent off-label use of contrast agents and the CEUS examination is performed under the responsibility of the physician. The benefit of reducing ionizing radiation is obviously most important in children and fertile women, but should always be weighed against its possible disadvantages in the actual clinical context of the individual patient. This important question has been discussed in the literature $[43,44]$, and while there is no easy way out of this medico-legal dilemma, it should always be borne in mind that the physician's top priority must be the patient's well-being. For reasons that are not always clear, children admitted acutely in the middle of the night with abdominal pain may sometimes undergo a CT scan to rule out serious conditions that would require surgery, which may result in the diagnosis of an incidental lesion that eventually requires further work-up in the morning. Incidentally, detected focal splenic lesions are one such clinical scenario that is frequently encountered and represents a particular dilemma. Tumors of the spleen are rare findings, and although most of these lesions are hemangiomas or hamartomas, their differentiation from malignant tumors must be confirmed before they can be disregarded. The spleen has a similar sonographic appearance to the liver and this fact sometimes brings about the misperception that focal spleen lesions have similar contrast uptake to FLLs and can therefore be interpreted according to the algorithm used for the liver (Table 1). Unfortunately, this is not the case. Although the spleen, like the liver, actually does have sinusoids, a very important difference from the liver is that the spleen has no equivalent to the portal vein and thus no dual blood

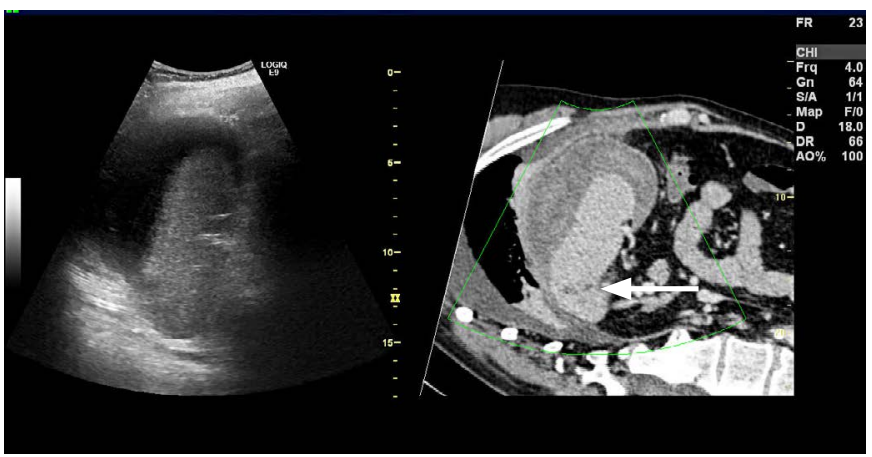

A

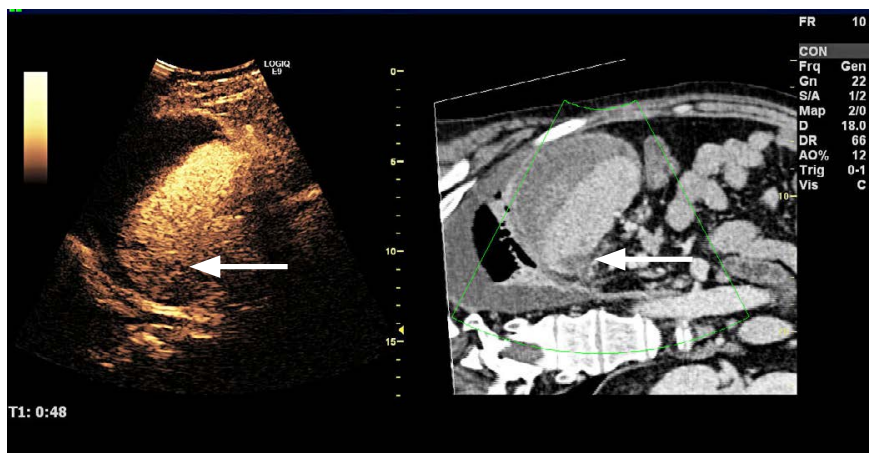

B

Fig. 4. A 50-year-old man with intrasplenic hematoma and a partial rupture with a large perisplenic hematoma and fluid collection after a car accident, visualized with contrast-enhanced computed tomography (CECT).

Two days later, the patient was in stable condition and it was decided to substitute contrast-enhanced ultrasonography (CEUS) examination in place of CECT for follow-up. CEUS was performed with SonoVue and previously captured CECT scan was fused with the present CEUS study. A. Ultrasonography-computed tomography (CT) fused image at a follow-up 2 days after the accident shows the perisplenic fluid collection with a similar size as found in the CT 2 days previously. In addition, a minor laceration (arrow) is present in the spleen parenchyma near the medial-cranial pole, which is not as clearly visualized in the sonogram (left side) as in the corresponding fused CT image (right side). B. Fused CEUS and CT clearly demonstrates the small laceration (arrows), and also confirms that there is no extravasation of ultrasound contrast agent, thus demonstrating that bleeding from the spleen had ceased. This figure illustrates the utility of CEUS as substitute for CECT scans in follow-up after major trauma. 
supply. Further complicating matters, unfortunately, benign spleen lesions frequently show wash-out in the parenchymal phase, and the fact that this is a major indicator of malignancy in the work-up of FLLs may lead to the false-positive diagnosis of a malignant splenic tumor $[45,46]$. If presented with an otherwise healthy patient with a rare incidental finding that is most often benign but may lead to an erroneous diagnosis of malignancy on CEUS, it would seem prudent to perform a follow-up CEUS one and three months later. If malignancy still cannot be ruled out with certainty, positron emission tomography-CT has been suggested as a definitive step [47] before performing ultrasound-guided biopsy with its low but not negligible risk. Fig. 5 (with Video clips 11, 12) demonstrates the clinical problem of the false-positive CEUS diagnosis of a malignant spleen lesion based on parenchymal hypoenhancement. The final diagnosis, verified by an ultrasound-guided biopsy, was the benign finding of a hamartoma.

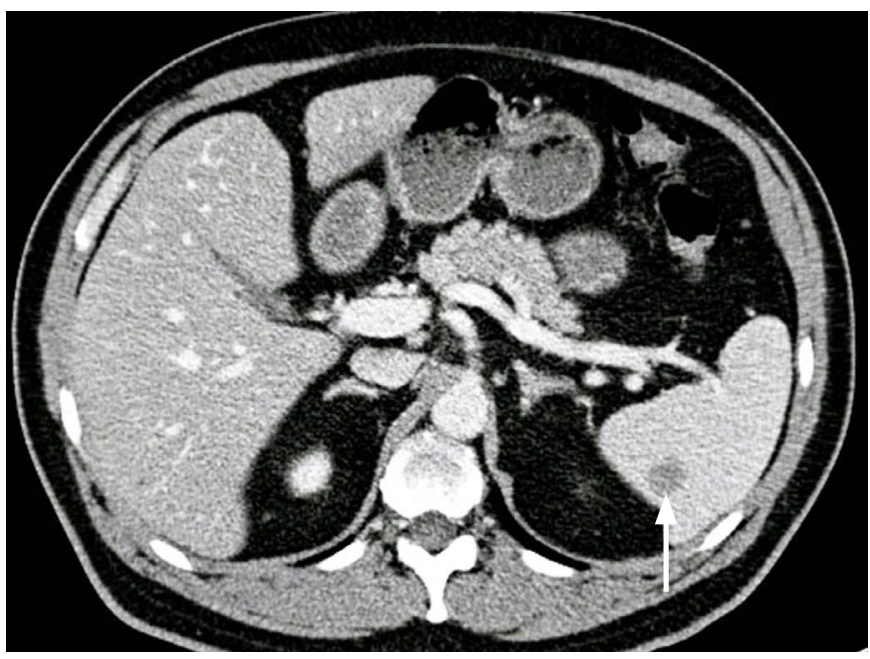

A

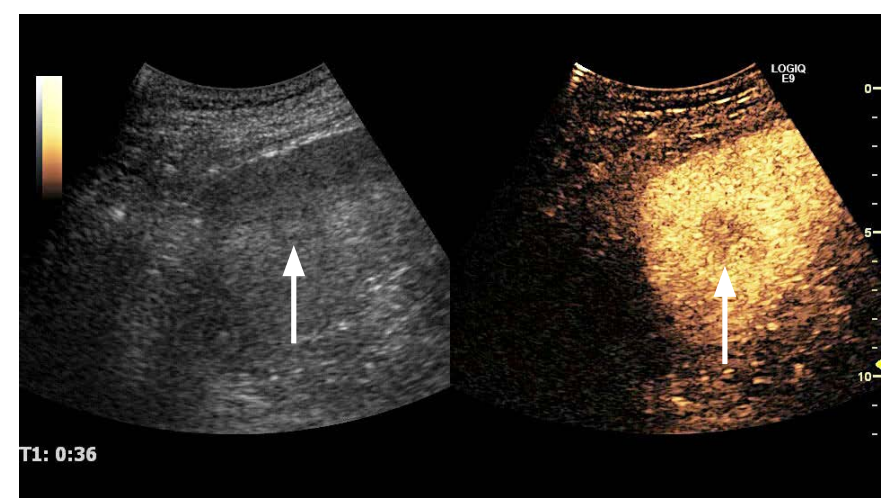

C

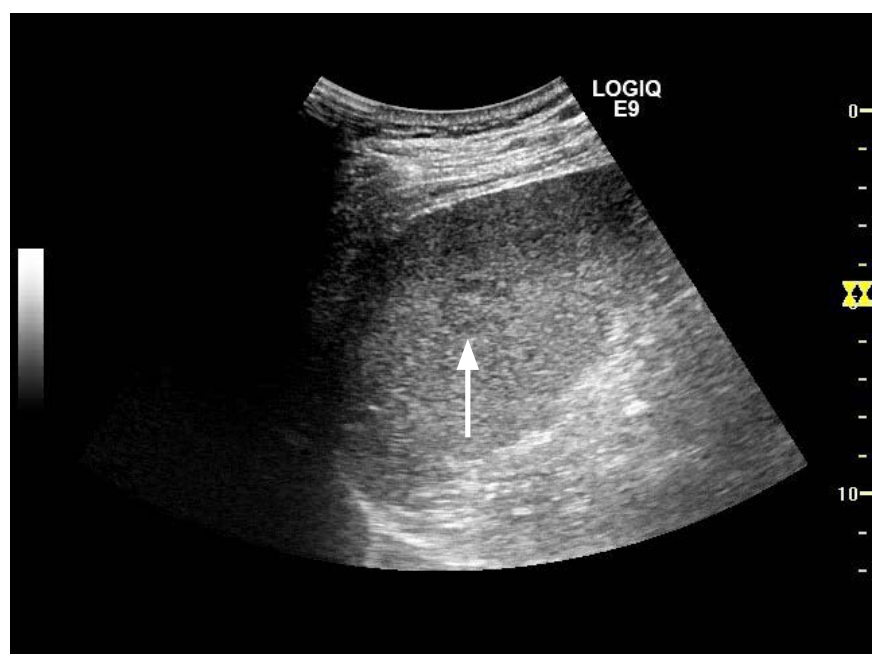

B

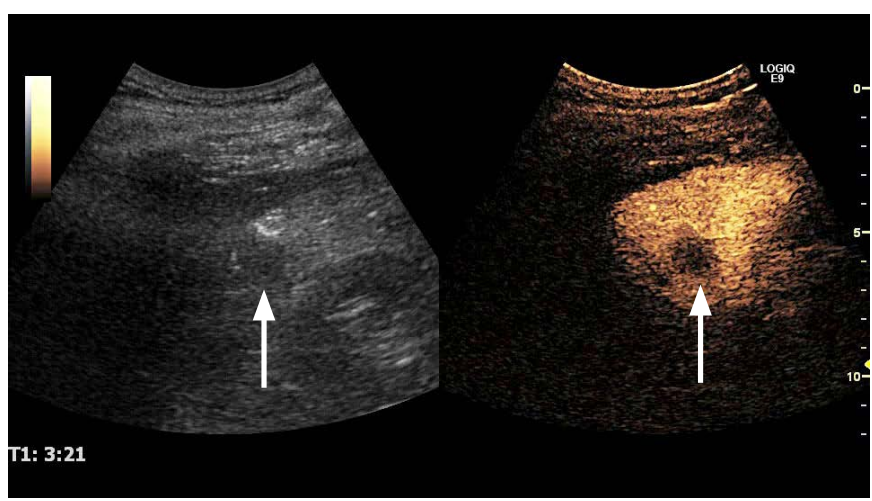

D

Fig. 5. A 57-year-old man with abdominal pain with coincidental focal lesion of the spleen diagnosed on contrast-enhanced computed tomography (CECT).

The patient had no previous history of cancer. However, in a subsequent work-up, contrast-enhanced ultrasonography (CEUS) found the lesion suspicious and the local tumor board decided to refer the patient to our unit for a percutaneous biopsy to rule out malignancy. A. CECT from the referring tumor board shows a focal hypodense spleen lesion (arrow). B. B-mode ultrasonography performed in our unit demonstrates a slightly hypoechoic lesion (arrow) in the spleen. C. CEUS during the arterial phase demonstrates enhancement of the lesion, which is slightly delayed and hypoenhancing (arrows) compared to the surrounding normal spleen tissue. D. CEUS demonstrates strong hypoenhancement (arrows), also known as wash-out, in the late parenchymal phase. From CEUS-guided biopsy, the final diagnosis was splenoma, which is a benign hamartoma-like tumor of the spleen. In conclusion, CEUS wash-out is not indicative of a strong suspicion of malignancy, and a clinical examination and positron emission tomography-computed tomography should be considered before biopsy in patients with no other indications of malignant disease. This figure illustrates that wash-out in spleen lesion CEUS is not equivalent to the CEUS of focal liver lesions and cannot be taken as a strong indicator of malignancy. 


\section{Guidelines and Good Clinical Practice} Recommendations for CEUS in the Liver: Update 2012: A WFUMB-EFSUMB Initiative in Cooperation with Representatives of AFSUMB, AIUM, ASUM, FLAUS and ICUS

This third generation of CEUS liver guidelines was published simultaneously in the February 2013 issues of Ultrasound in Medicine and Biology and European Journal of U/trasound/UItraschall in der Medizin $[3,4]$. The key importance of this document is the fact it presented a worldwide consensus from a wide and truly representative range of medical professional bodies on the usage of CEUS and the interpretation of enhancement patterns with clinically approved contrast agents for liver applications. The article describes the use of available different contrast agents licensed across the world for use in the work-up of clinical scenarios related to the liver.

These contrast agents are all fluorocarbons, sometimes referred to as perfluorocarbons, and also sometimes called organofluorine compounds. They are biologically inert gases at atmospheric pressure that are encapsulated in a lipid membranous shell. Thus, they do not interact with metabolism, can be administered regardless of renal and liver functionality, and have an extremely high safety profile, as specifically documented for SonoVue [24] (marketed as Lumason in the United States but not licensed for the liver), which is a sulfur hexafluoride introduced in 2001 by Bracco SpA (Milan, Italy) and licensed in Europe, China, India, Korea, Hong Kong, New Zealand, Singapore, and Brazil. The other two contrast agents are chemically very closely related and marketed under the names Definity/Luminity,

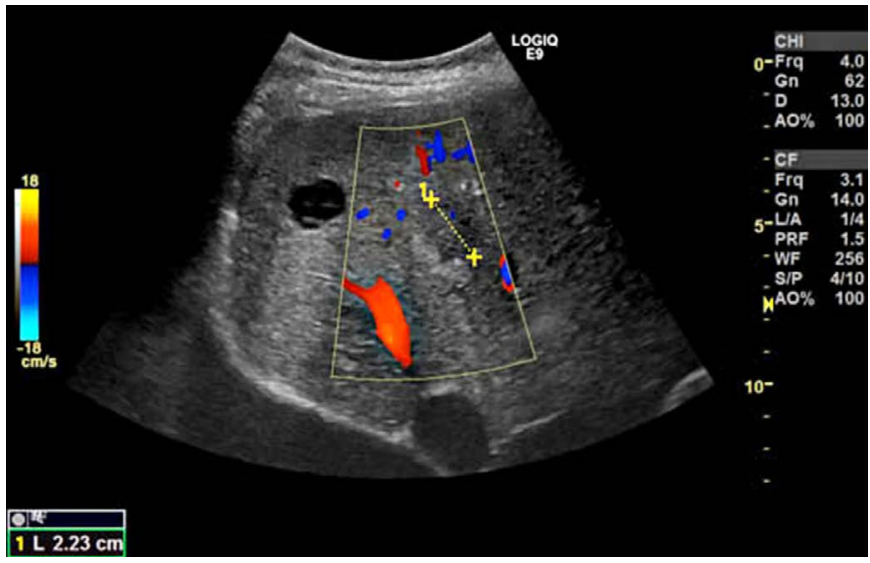

A

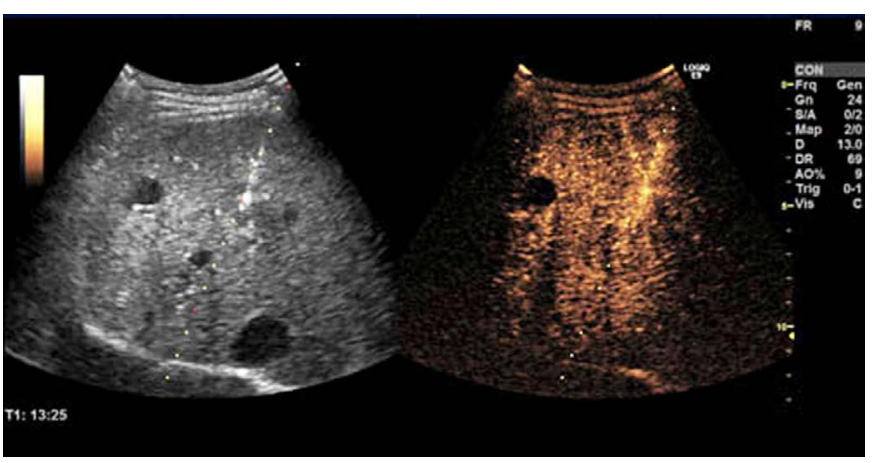

C

Fig. 6. A 77-year-old woman under surveillance for cirrhosis.

A. B-mode and color Doppler ultrasonography demonstrate an interim nodule (crosshairs) in the right hepatic lobe. B. Sonazoid contrastenhanced ultrasonography (CEUS) shows arterial hyper-enhancement (arrows) with earlier onset than the surrounding liver tissue. C. Sonazoid CEUS-guided 1.2-mm TruCut biopsy of an isoenhancing lesion in the postvascular phase was performed at 13 minutes post-contrast. The biopsy revealed well-differentiated hepatocellular carcinoma. D. An image from a Sonazoid CEUS shows sustained isoenhancement in the postvascular phase at 31 minutes post-contrast. This figure illustrates the fact that with Sonazoid CEUS of welldifferentiated hepatocellular carcinomas, in approximately $50 \%$ of cases, no enhancement defect is noted at 30 minutes post-contrast, potentially warranting a biopsy. 
which is an octafluoropropane compound introduced in 2001 by Lantheus Medical (Billerica, MA, USA) and licensed in Canada and Australia, and Sonazoid, a perfluorobutane introduced in 2007 by Daiichi-Sankyo (GE, Tokyo, Japan) and licensed in Japan and South Korea, and recently also in Denmark and Norway.

A search on Scopus on August 28, 2015 showed that the 2012 update of the liver CEUS guidelines had been cited a total of 170 times in 97 scientific journals, with 80 citations in 52 journals of the version of the article printed in Ultrasound in Medicine and Biology and 90 citations in 45 journals of the version printed in European Journal of Ultrasound/UItraschall in der Medizin. Compared to the two previous generations of CEUS guidelines, this most recent update provided recommendations on two new topics: information related to Sonazoid and a recommendation to use CEUS for biopsy guidance to increase the retrieval rate of percutaneous ultrasoundguided biopsy and to reduce the frequency of negative microscopy results [48-52].

Sonazoid, remarkably and completely unlike the other two contrast agents, has a so-called postvascular phase in which the contrast agent leaves the vascular bed and enters the parenchymal interstitium. This postvascular phase starts 10 minutes after injection and may last more than 30 minutes, and is caused by the Kupffer cell uptake of this particular contrast agent, which is very similar to that of the liver-specific MRI contrast agents often referred to as superparamagnetic iron oxide (SPIO) [53]. This mechanism ensures the uniform enhancement of normal liver tissue. Sonazoid, according to Kudo et al. [54-56] has been used with particular frequency in the work-up of hepatocellular carcinoma (HCC) in the cirrhotic liver because the typical HCC contains no Kupffer cells and thus stands out as an enhancement defect in the postvascular phase [57]. Unfortunately, however, between $50 \%$ and $70 \%$ of well differentiated HCCs-corresponding to approximately $10 \%-15 \%$ of HCCs in the cirrhotic population-may not demonstrate this typical enhancement defect $[58,59]$, and in this situation a biopsy may be indicated to achieve the correct diagnosis $[56,60,61]$. Fig. 6 (with Video clips 13-15) demonstrates a clinical scenario such as this, in which a CEUS-guided biopsy was performed utilizing Sonazoid. The benefit of CEUS-guided biopsy was reported by Bang et al. [50] as early as 2000 and later was proven by Wu et al. [49] to increase the diagnostic accuracy rate by up to $10 \%$ by directing the biopsy towards contrast-enhanced areas. Schlottman et al. [48] suggested a different approach, in which CEUS was used to identify previously non-visualized lesions that were more easily accessible for biopsy, and this technique later received support from Yoon et al. [51,52].

In addition to the work-up of liver lesions, which has been described in detail above, the single other major indication for CEUS that has existed since the initial development of this technique is assistance in percutaneous ablative procedures. In many institutions around the world, ultrasonography is the preferred guidance modality for ablation. However, with regard to treatment evaluation, it is not sufficient to examine morphology alone, since tissue viability cannot be judged without knowledge of perfusion, which necessitates the use of contrast agents. Additionally, the ablation volume itself occasionally may present on B-mode ultrasonography very similarly to the surrounding normal tissue, again making the use of contrast agents necessary. Thus, before the availability of CEUS,

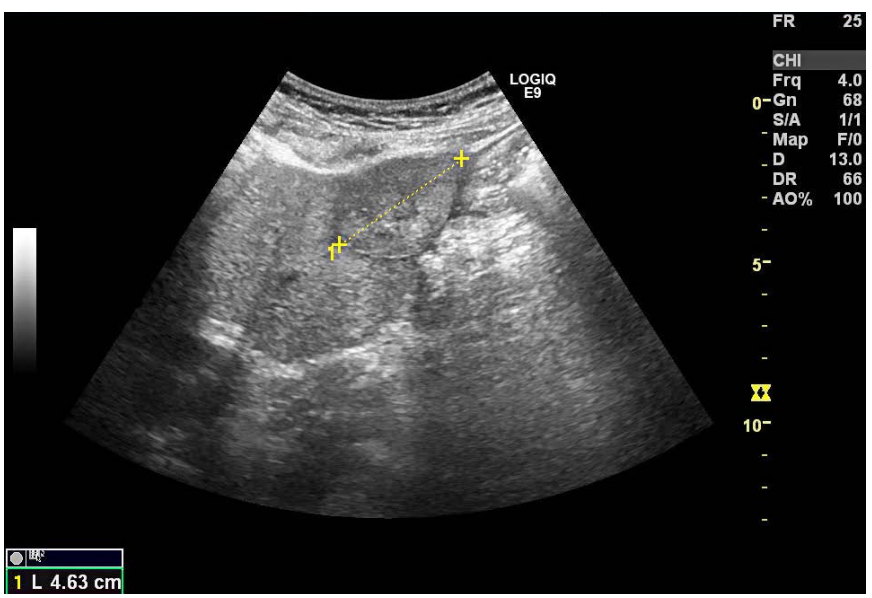

A

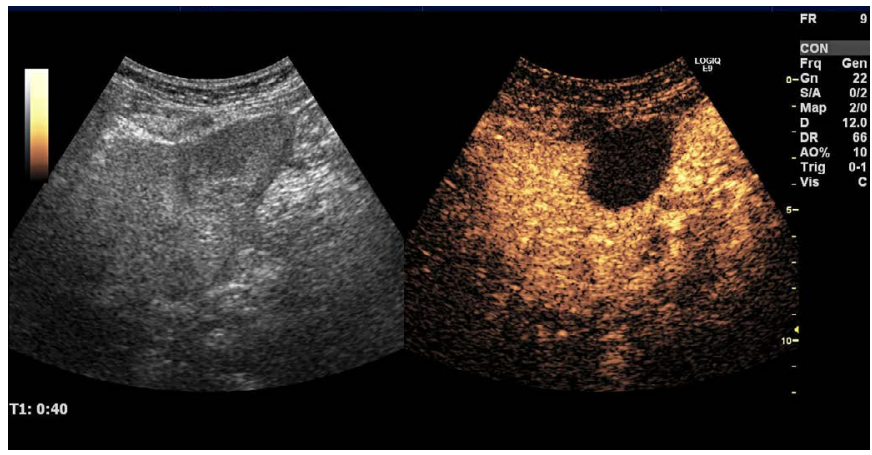

B

Fig. 7. Contrast-enhanced ultrasonography (CEUS) assistance during the course of follow-up after percutaneous ablation.

A. On B-mode ultrasonography, the ablation volume (crosshairs) cannot be evaluated for viability on 6-week follow-up after the microwave ablation of a solitary colorectal liver metastasis. B. SonoVue CEUS shows a well-demarcated ablation cavity without any enhancement, indicating successful ablation with no suspicion of skipped areas and no viable tissue. 


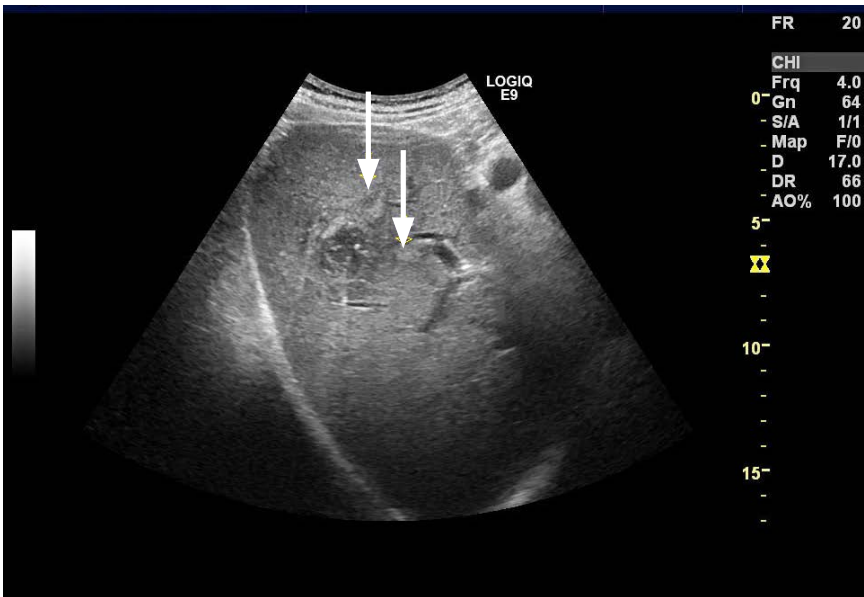

A

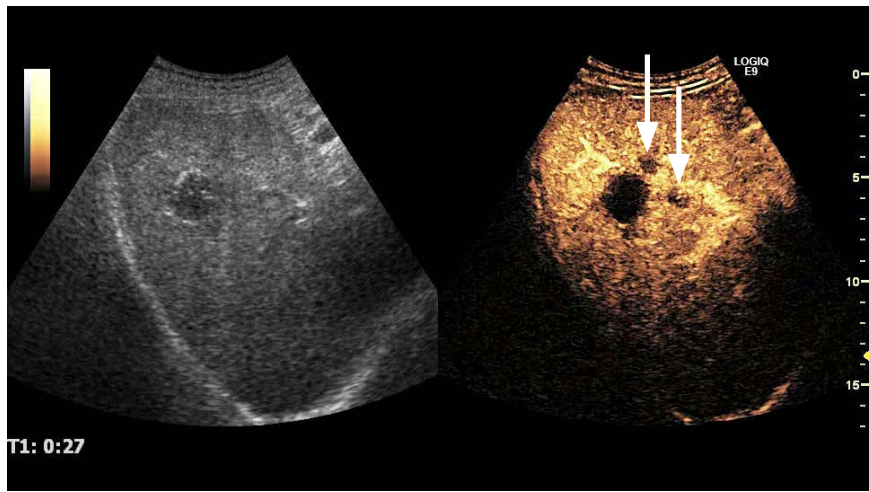

B

Fig. 8. Contrast-enhanced ultrasonography (CEUS) assistance during the course of follow-up after percutaneous ablation with CEUSguided biopsy for suspicious local recurrence.

A. One-year follow-up B-mode ultrasonography after the radiofrequency ablation of hepatocellular carcinoma (HCC) indicates the suspicion of a local recurrence (arrows). B. SonoVue CEUS well delineates the suspicious recurrent lesions (arrows) more clearly. After reinjection of SonoVue, a CEUS-guided cytology biopsy was directed towards the suspected recurrence area, and microscopy confirmed the diagnosis of local recurrence of HCC.

it was mandatory to include CT or MRI in the follow-up protocol of ablation therapy. With the advent of CEUS, ultrasonography can play a pivotal role in all aspects of percutaneous ablation from diagnosis, through guidance and procedural monitoring [62-64], to follow-up examinations after percutaneous ablation. Recent reports, including one multicenter study, have concluded that CEUS is comparable in performance to both contrast-enhanced CT and MRI $[65,66]$ in the evaluation of ablation treatment for local recurrence, the assessment of ablation volume viability, and the identification of new metastases. Fig. 7 (with Video clip 16) and Fig. 8 (with Video clip 17) illustrate how CEUS is an indispensable tool in ultrasonography follow-up after percutaneous ablation, both with respect to imaging and as guidance for percutaneous biopsy in the case of relapse.

\section{Conclusion}

The introduction of contrast agents for ultrasonography has had an overwhelming impact on modern medicine. CEUS has dramatically expanded the field of medical ultrasound and opened up a completely new world in patient management. In liver imaging, CEUS has come to play a decisive role, due to its excellent temporal resolution, and the use of CEUS has changed the classic indications for biopsies and limited biopsy to equivocal cases. CEUS in many aspects is comparable to contrast-enhanced CT and MRI [1 $4,10,15-19,22,23,65]$, and CEUS has changed the algorithms for medical imaging in high-income industrialized societies. According to the WHO, two thirds of the world's population has no access to medical imaging [6]. Ultrasonography including CEUS has the potential to rectify this inequality, and if CEUS were incorporated as an integral part of ultrasonography, it would be realistic to bring state-of-the-art medical imaging to the entire world. Just imagine how the lack of availability of contrast agents would limit the value and reduce the impact of CT or MRI! This is the scenario ultrasonography must face in many countries where contrast agents have not been approved. The World Federation for Ultrasound in Medicine and Biology has as its overall purpose to bring the use of medical ultrasound to every corner of the world. Used together with all its technical refinements, including CEUS, ultrasonography is a second-to-none multipotent imaging tool capable of providing state-of-the-art imaging to the entire world in the new millennium.

ORCID: Christian Pállson Nolsøe: http://orcid.org/0000-0003-0168-6131

Abbreviations: AFSUMB, Asian Federation of Societies for Ultrasound in Medicine and Biology; AIUM, American Institute of Ultrasound in Medicine; ASUM, Australasian Society for Ultrasound in Medicine; CEUS, contrast-enhanced ultrasonography; CT, computed tomography; EFSUMB, European Federation of Societies for Ultrasound in Medicine and Biology; FLAUS, Latin American Federation of Societies for Ultrasound in Medicine and Biology; FLL, focal liver lesion; HCC, hepatocellular carcinoma; ICUS, International Contrast Ultrasound Society; MRI, magnetic resonance imaging; 
WFUMB, World Federation for Ultrasound in Medicine and Biology; WHO, World Health Organization.

\section{Conflict of Interest}

No potential conflict of interest relevant to this article was reported.

\section{Supplementary Material}

Video clip 1. In the arterial phase, the hyperechoic lesion shows globular filling-in contrast enhancement, whereas the hypoechoic lesion demonstrated arterial hyperenhancement (http://dx.doi. org/10.14366/usg.15057.v001).

Video clip 2. During the portal phase, the hyperechoic lesion continues the same pattern of contrast filling-in enhancement, whereas the hypoechoic lesion shows a contrast wash-out pattern, thus confirming the B-mode diagnosis of hemangioma and metastasis (http://dx.doi.org/10.14366/usg.15057.v002).

Video clip 3. During the late phase, the same contrast enhancement pattern as in Video clip 2C continues (http://dx.doi.org/10.14366/ usg. 15057.v003).

Video clip 4. Contrast-enhanced ultrasonography sequence illustrates arterial contrast uptake with very early (before normal liver tissue) enhancement of the central lesion with a centrifugal pattern, a feeding artery consistent with the focal nodular hyperplasia arterial pattern, and the slower centripetal globular filling-in enhancement pattern of the larger lesion consistent with the arterial pattern of hemangioma (http://dx.doi.org/10.14366/usg.15057.v004).

Video clip 5. Contrast-enhanced ultrasonography sequence captured immediately after Video clip 4, illustrates arterial contrast uptake with slow centripetal globular filling-in enhancement pattern of another more peripherally located lesion consistent with the arterial pattern of hemangioma (http://dx.doi.org/10.14366/ usg.15057.v005).

Video clip 6. Contrast-enhanced ultrasonography from the portal phase shows the typical slow filling-in of a large hemangioma in which the central areas do not display enhancement, likely due to necrosis and scarring, as well as the sustained hyperenhancement of focal nodular hyperplasia (http://dx.doi.org/10.14366/usg.15057. v006).

Video clip 7. A contrast-enhanced ultrasonography sequence in the parenchymal phase shows the typical slow filling-in of a large hemangioma in which the central areas do not display enhancement, as well as the sustained hyperenhancement of focal nodular hyperplasia, with both features extending long into the parenchymal phase (http://dx.doi.org/10.14366/usg.15057.v007).

Video clip 8. In a patient of minor spleen trauma, contrastenhanced ultrasonography of the left flank clearly shows a small hematoma in the lower pole of the spleen (http://dx.doi. org/10.14366/usg.15057.v008).

Video clip 9. Ultrasonography-computed tomography (CT) fusion at follow-up two days after the accident shows the perisplenic fluid collection with a similar size as found in the CT imaging two days previously. In addition, a minor laceration is present in the spleen parenchyma near the medial-cranial pole, which is not as clearly visualized in the sonogram as in the corresponding fused CT (http://dx.doi.org/10.14366/usg.15057.v009).

Video clip 10. A fused contrast-enhanced ultrasonography and computed tomography image clearly demonstrates the small laceration, and also confirms that no extravasation of the ultrasound contrast agent was present, thus demonstrating that bleeding from the spleen had ceased (http://dx.doi.org/10.14366/usg.15057. v010).

Video clip 11. A contrast-enhanced sonogram in the arterial phase performed in our unit demonstrates arterial enhancement, which is slightly delayed and slightly hypoenhancing compared to the surrounding normal spleen tissue (http://dx.doi.org/10.14366/ usg.15057.v011).

Video clip 12. By a contrast-enhanced ultrasonography-guided biopsy, the final diagnosis was splenoma, a benign hamartoma-like tumor of the spleen (http://dx.doi.org/10.14366/usg.15057.v012).

Video clip 13. A Sonazoid contrast-enhanced ultrasonography shows arterial hyperenhancement with earlier onset than the surrounding liver tissue (please note that there is in addition a simple cyst in the patients right upper corner of the Video clip which appears typically totally non-enhancing and echopoor) (http://dx.doi.org/10.14366/usg.15057.v013).

Video clip 14. A Sonazoid contrast-enhanced ultrasonongraphyguided 1.2-mm TruCut biopsy of an isoenhancing lesion in the postvascular phase at 13 minutes post-contrast revealed welldifferentiated hepatocellular carcinoma (http://dx.doi.org/10.14366/ usg.15057.v014). 
Video clip 15. A Sonazoid contrast-enhancing ultrasonography shows sustained isoenhancement in the postvascular phase at 31 minutes post-contrast (http://dx.doi.org/10.14366/usg.15057. v015).

Video clip 16. SonoVue contrast-enhanced ultrasonography shows a well-demarcated ablation cavity without any enhancement, indicating successful ablation with no suspicion of skipped areas and no viable tissue (http://dx.doi.org/10.14366/usg.15057.v016).

Video clip 17. After the reinjection of SonoVue, a contrastenhanced ultrasonography-guided cytology biopsy was directed towards the suspected recurrence area, and microscopy confirmed the diagnosis of local recurrence (http://dx.doi.org/10.14366/ usg.15057.v017).

\section{References}

1. Albrecht T, Blomley M, Bolondi L, Claudon M, Correas JM, Cosgrove $D$, et al. Guidelines for the use of contrast agents in ultrasound. January 2004. Ultraschall Med 2004;25:249-256.

2. Claudon M, Cosgrove D, Albrecht T, Bolondi L, Bosio M, Calliada F, et al. Guidelines and good clinical practice recommendations for contrast enhanced ultrasound (CEUS): update 2008. Ultraschall Med 2008;29:28-44.

3. Claudon M, Dietrich CF, Choi BI, Cosgrove DO, Kudo M, Nolsoe CP, et al. Guidelines and good clinical practice recommendations for contrast enhanced ultrasound (CEUS) in the liver: update 2012: a WFUMB-EFSUMB initiative in cooperation with representatives of AFSUMB, AIUM, ASUM, FLAUS and ICUS. Ultraschall Med 2013;34:11-29.

4. Claudon M, Dietrich CF, Choi BI, Cosgrove DO, Kudo M, Nolsoe CP, et al. Guidelines and good clinical practice recommendations for Contrast Enhanced Ultrasound (CEUS) in the liver: update 2012. A WFUMB-EFSUMB initiative in cooperation with representatives of AFSUMB, AIUM, ASUM, FLAUS and ICUS. Ultrasound Med Biol 2013;39:187-210.

5. Piscaglia F, Nolsoe C, Dietrich CF, Cosgrove DO, Gilja OH, Bachmann Nielsen $\mathrm{M}$, et al. The EFSUMB Guidelines and Recommendations on the Clinical Practice of Contrast Enhanced Ultrasound (CEUS): update 2011 on non-hepatic applications. Ultraschall Med 2012;33:33-59.

6. Hussain S. Welcome to the Journal of Global Radiology. J Glob Radiol 2015;1:1.

7. Sidhu PS. Multiparametric ultrasound (MPUS) imaging: terminology describing the many aspects of ultrasonography. Ultraschall Med 2015:36:315-317.

8. Volk M, Strotzer M, Lenhart M, Techert J, Seitz J, Feuerbach S.
Frequency of benign hepatic lesions incidentally detected with contrast-enhanced thin-section portal venous phase spiral CT. Acta Radiol 2001;42:172-175.

9. Little JM, Richardson A, Tait N. Hepatic dystychoma: a five year experience. HPB Surg 1991;4:291-297.

10. Strobel D, Seitz K, Blank W, Schuler A, Dietrich C, von Herbay A, et al. Contrast-enhanced ultrasound for the characterization of focal liver lesions: diagnostic accuracy in clinical practice (DEGUM multicenter trial). Ultraschall Med 2008;29:499-505.

11. Bernatik T, Seitz K, Blank W, Schuler A, Dietrich CF, Strobel D. Unclear focal liver lesions in contrast-enhanced ultrasonography: lessons to be learned from the DEGUM multicenter study for the characterization of liver tumors. Ultraschall Med 2010;31:577-581.

12. Seitz K, Bernatik T, Strobel D, Blank W, Friedrich-Rust M, Strunk H, et al. Contrast-enhanced ultrasound (CEUS) for the characterization of focal liver lesions in clinical practice (DEGUM Multicenter Trial): CEUS vs. MRI: a prospective comparison in 269 patients. Ultraschall Med 2010;31:492-499.

13. Seitz K, Strobel D, Bernatik T, Blank W, Friedrich-Rust M, Herbay A, et al. Contrast-Enhanced Ultrasound (CEUS) for the characterization of focal liver lesions: prospective comparison in clinical practice: CEUS vs. CT (DEGUM multicenter trial). Parts of this manuscript were presented at the Ultrasound Dreilandertreffen 2008, Davos. Ultraschall Med 2009;30:383-389.

14. Strobel D, Bernatik T, Blank W, Schuler A, Greis C, Dietrich CF, et al. Diagnostic accuracy of CEUS in the differential diagnosis of small $(\leq 20 \mathrm{~mm})$ and subcentimetric $(\leq 10 \mathrm{~mm})$ focal liver lesions in comparison with histology. Results of the DEGUM multicenter trial. Ultraschall Med 2011;32:593-597.

15. Trillaud H, Bruel JM, Valette PJ, Vilgrain V, Schmutz G, Oyen R, et al. Characterization of focal liver lesions with SonoVue-enhanced sonography: international multicenter-study in comparison to CT and MRI. World J Gastroenterol 2009;15:3748-3756.

16. Tranquart F, Correas JM, Ladam Marcus V, Manzoni P, Vilgrain $V$, Aube $C$, et al. Real-time contrast-enhanced ultrasound in the evaluation of focal liver lesions: diagnostic efficacy and economical issues from a French multicentric study. J Radiol 2009;90(1 Pt 2):109-122.

17. Guang Y, Xie L, Ding H, Cai A, Huang Y. Diagnosis value of focal liver lesions with SonoVue(R)-enhanced ultrasound compared with contrast-enhanced computed tomography and contrast-enhanced MRI: a meta-analysis. J Cancer Res Clin Oncol 2011;137:15951605.

18. Xie L, Guang Y, Ding H, Cai A, Huang Y. Diagnostic value of contrast-enhanced ultrasound, computed tomography and magnetic resonance imaging for focal liver lesions: a meta-analysis. Ultrasound Med Biol 2011;37:854-861.

19. Westwood M, Joore M, Grutters J, Redekop K, Armstrong N, Lee $\mathrm{K}$, et al. Contrast-enhanced ultrasound using SonoVue(R) (sulphur 
hexafluoride microbubbles) compared with contrast-enhanced computed tomography and contrast-enhanced magnetic resonance imaging for the characterisation of focal liver lesions and detection of liver metastases: a systematic review and cost-effectiveness analysis. Health Technol Assess 2013;17:1-243.

20. D'Onofrio M, Crosara S, De Robertis R, Canestrini S, Mucelli RP. Contrast-enhanced ultrasound of focal liver lesions. AJR Am J Roentgenol 2015;205:W56-W66.

21. Dietrich CF, Sharma M, Gibson RN, Schreiber-Dietrich D, Jenssen C. Fortuitously discovered liver lesions. World I Gastroenterol 2013;19:3173-3188.

22. Cantisani V, Grazhdani H, Fioravanti C, Rosignuolo M, Calliada F, Messineo D, et al. Liver metastases: contrast-enhanced ultrasound compared with computed tomography and magnetic resonance. World J Gastroenterol 2014;20:9998-10007.

23. Friedrich-Rust M, Klopffleisch T, Nierhoff J, Herrmann E, Vermehren J, Schneider MD, et al. Contrast-enhanced ultrasound for the differentiation of benign and malignant focal liver lesions: a metaanalysis. Liver Int 2013;33:739-755.

24. Piscaglia F, Bolondi L; Italian Society for Ultrasound in Medicine and Biology (SIUMB) Study Group on Ultrasound Contrast Agents. The safety of Sonovue in abdominal applications: retrospective analysis of 23188 investigations. Ultrasound Med Biol 2006;32:1369-1375.

25. Nolsoe CP, Lorentzen T, Skjoldbye BO, Bachmann Nielsen M. The basics of interventional ultrasound. Ultraschall Med 2007;28:248263.

26. Karhunen PJ. Benign hepatic tumours and tumour like conditions in men. J Clin Pathol 1986;39:183-188.

27. Dietrich CF, Mertens JC, Braden B, Schuessler G, Ott M, Ignee A. Contrast-enhanced ultrasound of histologically proven liver hemangiomas. Hepatology 2007;45:1139-1145.

28. Catalano O, Lobianco R, Sandomenico F, Siani A. Splenic trauma: evaluation with contrast-specific sonography and a secondgeneration contrast medium: preliminary experience. J Ultrasound Med 2003;22:467-477.

29. McGahan JP, Horton S, Gerscovich EO, Gillen M, Richards JR, Cronan MS, et al. Appearance of solid organ injury with contrastenhanced sonography in blunt abdominal trauma: preliminary experience. AJR Am J Roentgenol 2006;187:658-666.

30. Thorelius L. Contrast-enhanced ultrasound in trauma. Eur Radiol 2004;14 Suppl 8:P43-P52.

31. Afaq A, Harvey C, Aldin Z, Leen E, Cosgrove D. Contrast-enhanced ultrasound in abdominal trauma. Eur J Emerg Med 2012;19:140145.

32. Catalano O, Sandomenico F, Matarazzo I, Siani A. Contrastenhanced sonography of the spleen. AJR Am J Roentgenol 2005; 184:1150-1156.

33. Clevert DA, Weckbach $S$, Minaifar N, Clevert DA, Stickel M, Reiser M. Contrast-enhanced ultrasound versus MS-CT in blunt abdominal trauma. Clin Hemorheol Microcirc 2008;39:155-169.

34. Cokkinos D, Antypa E, Stefanidis K, Tserotas P, Kostaras V, Parlamenti $A$, et al. Contrast-enhanced ultrasound for imaging blunt abdominal trauma: indications, description of the technique and imaging review. Ultraschall Med 2012;33:60-67.

35. Lv F, Ning Y, Zhou $X$, Luo $Y$, Liang T, Nie $Y$, et al. Effectiveness of contrast-enhanced ultrasound in the classification and emergency management of abdominal trauma. Eur Radiol 2014;24:26402648.

36. Lv F, Tang J, Luo Y, Li Z, Meng X, Zhu Z, et al. Contrast-enhanced ultrasound imaging of active bleeding associated with hepatic and splenic trauma. Radiol Med 2011;116:1076-1082.

37. Mihalik JE, Smith RS, Toevs CC, Putnam AT, Foster JE. The use of contrast-enhanced ultrasound for the evaluation of solid abdominal organ injury in patients with blunt abdominal trauma. J Trauma Acute Care Surg 2012;73:1100-1105.

38. Pinto F, Miele V, Scaglione M, Pinto A. The use of contrast-enhanced ultrasound in blunt abdominal trauma: advantages and limitations. Acta Radiol 2014;55:776-784.

39. Pinto F, Valentino M, Romanini L, Basilico R, Miele V. The role of CEUS in the assessment of haemodynamically stable patients with blunt abdominal trauma. Radiol Med 2015;120:3-11.

40. Sessa B, Trinci M, Ianniello S, Menichini G, Galluzzo M, Miele V. Blunt abdominal trauma: role of contrast-enhanced ultrasound (CEUS) in the detection and staging of abdominal traumatic lesions compared to US and CE-MDCT. Radiol Med 2015;120:180-189.

41. Catalano O, Aiani L, Barozzi L, Bokor D, De Marchi A, Faletti C, et al. CEUS in abdominal trauma: multi-center study. Abdom Imaging 2009;34:225-234.

42. Menichini G, Sessa B, Trinci M, Galluzzo M, Miele V. Accuracy of contrast-enhanced ultrasound (CEUS) in the identification and characterization of traumatic solid organ lesions in children: a retrospective comparison with baseline US and CE-MDCT. Radiol Med 2015;120:989-1001.

43. Piskunowicz M, Kosiak W, Irga N. Primum non nocere? Why can't we use second generation ultrasound contrast agents for the examination of children? Ultraschall Med 2011;32:83-86.

44. Schreiber-Dietrich DG, Cui XW, Piscaglia F, Gilja OH, Dietrich CF. Contrast enhanced ultrasound in pediatric patients: a real challenge. Z Gastroenterol 2014;52:1178-1184.

45. Stang A, Keles H, Hentschke S, von Seydewitz CU, Dahlke J, Habermann $C$, et al. Incidentally detected splenic lesions in ultrasound: does contrast-enhanced ultrasonography improve the differentiation of benign hemangioma/hamartoma from malignant lesions? Ultraschall Med 2011;32:582-592.

46. Yu X, Yu J, Liang P, Liu F. Real-time contrast-enhanced ultrasound in diagnosing of focal spleen lesions. Eur J Radiol 2012;81:430-436.

47. Metser U, Miller E, Kessler A, Lerman H, Lievshitz G, Oren R, et al. Solid splenic masses: evaluation with 18F-FDG PET/CT. J Nucl Med 
2005;46:52-59.

48. Schlottmann K, Klebl F, Zorger N, Feuerbach S, Scholmerich J. Contrast-enhanced ultrasound allows for interventions of hepatic lesions which are invisible on convential B-mode. Z Gastroenterol 2004;42:303-310.

49. Wu W, Chen MH, Yin SS, Yan K, Fan ZH, Yang W, et al. The role of contrast-enhanced sonography of focal liver lesions before percutaneous biopsy. AJR Am J Roentgenol 2006;187:752-761.

50. Bang N, Bachmann Nielsen M, Vejborg I, Mellon Mogensen A. Clinical report: contrast enhancement of tumor perfusion as a guidance for biopsy. Eur J Ultrasound 2000;12:159-161.

51. Sparchez Z, Radu P, Zaharia T, Kacso G, Grigorescu I, Botis G, et al. Usefulness of contrast enhanced ultrasound guidance in percutaneous biopsies of liver tumors. J Gastrointestin Liver Dis 2011;20:191-196.

52. Yoon SH, Lee KH, Kim SY, Kim YH, Kim JH, Lee SH, et al. Realtime contrast-enhanced ultrasound-guided biopsy of focal hepatic lesions not localised on B-mode ultrasound. Eur Radiol 2010;20:2047-2056.

53. Korenaga K, Korenaga M, Furukawa M, Yamasaki T, Sakaida I. Usefulness of Sonazoid contrast-enhanced ultrasonography for hepatocellular carcinoma: comparison with pathological diagnosis and superparamagnetic iron oxide magnetic resonance images. J Gastroenterol 2009;44:733-741.

54. Kudo M, Hatanaka K, Kumada T, Toyoda H, Tada T. Double-contrast ultrasound: a novel surveillance tool for hepatocellular carcinoma. Am J Gastroenterol 2011;106:368-370.

55. Kudo M, Hatanaka K, Maekawa K. Newly developed novel ultrasound technique, defect reperfusion ultrasound imaging, using sonazoid in the management of hepatocellular carcinoma. Oncology 2010;78 Suppl 1:40-45.

56. Kudo M, Matsui O, Izumi N, lijima H, Kadoya M, Imai Y, et al. Surveillance and diagnostic algorithm for hepatocellular carcinoma proposed by the Liver Cancer Study Group of Japan: 2014 update. Oncology 2014;87 Suppl 1:7-21.

57. Inoue T, Kudo M, Hatanaka K, Takahashi S, Kitai S, Ueda T, et al. Imaging of hepatocellular carcinoma: qualitative and quantitative analysis of postvascular phase contrast-enhanced ultrasonography with sonazoid. Comparison with superparamagnetic iron oxide magnetic resonance images. Oncology 2008;75 Suppl 1:48-54.

58. Arita J, Hasegawa K, Takahashi M, Hata S, Shindoh J, Sugawara $Y$, et al. Correlation between contrast-enhanced intraoperative ultrasound using Sonazoid and histologic grade of resected hepatocellular carcinoma. AJR Am J Roentgenol 2011;196:13141321.

59. Joo I, Choi BI. New paradigm for management of hepatocellular carcinoma by imaging. Liver Cancer 2012;1:94-109.

60. Kondo T, Maruyama H, Sekimoto T, Shimada T, Takahashi M, Chiba $\mathrm{T}$, et al. Natural history of postvascular-phase iso-enhanced lesions on the sonogram in chronic liver diseases. J Gastroenterol Hepatol 2014;29:165-172.

61. Zheng SG, Xu HX, Liu LN. Management of hepatocellular carcinoma: the role of contrast-enhanced ultrasound. World J Radiol 2014;6:7-14.

62. Liu F, Yu X, Liang P, Cheng Z, Han Z, Dong B. Contrast-enhanced ultrasound-guided microwave ablation for hepatocellular carcinoma inconspicuous on conventional ultrasound. Int J Hyperthermia 2011:27:555-562.

63. Lorentzen T, Skjoldbye BO, Nolsoe CP. Microwave ablation of liver metastases guided by contrast-enhanced ultrasound: experience with 125 metastases in 39 patients. Ultraschall Med 2011;32:492496.

64. Nishigaki Y, Hayashi H, Tomita E, Suzuki Y, Watanabe N, Watanabe $S$, et al. Usefulness of contrast-enhanced ultrasonography using Sonazoid for the assessment of therapeutic response to percutaneous radiofrequency ablation for hepatocellular carcinoma. Hepatol Res 2015;45:432-440.

65. Frieser $\mathrm{M}$, Kiesel J, Lindner A, Bernatik T, Haensler JM, Janka R, et al. Efficacy of contrast-enhanced US versus CT or MRI for the therapeutic control of percutaneous radiofrequency ablation in the case of hepatic malignancies. Ultraschall Med 2011;32:148-153.

66. Lu MD, Yu XL, Li AH, Jiang TA, Chen MH, Zhao BZ, et al. Comparison of contrast enhanced ultrasound and contrast enhanced CT or MRI in monitoring percutaneous thermal ablation procedure in patients with hepatocellular carcinoma: a multi-center study in China. Ultrasound Med Biol 2007;33:1736-1749. 